\title{
REAL TIME GESTURE MEASUREMENT OF HUMAN HAND FOR ROBOTICS ARM
}

\author{
R. Satheeshkumar ${ }^{1 *}$ and Dr. R. Arivoli ${ }^{2}$ \\ ${ }^{1}$ Research Scholar, Department of EEE, Annamalai University, Annamalai Nagar, \\ Tamilnadu, India \\ ${ }^{2}$ Professor, Department of EEE, Annamalai University, Annamalai Nagar, \\ Tamilnadu, India \\ ${ }^{1}$ satheesh2727@gmail.com, 2Inrarivolizc@yahoo.co.in
}

\begin{abstract}
In industry, Robotics needs precise control for handling various production activities. The proposed research is to measure the gesture grip value of both human hand and Robotic hand. The measured value of both human hand gripping and robotic hand gripping should be same. This leads for more perfection. Flex sensors are used to measure the value of gripping in both human hand and robotic hand. To achieve the exact control, camera visualizes the robotic hand activities from remote area. Pick and place of object is most significant task of robotic hand. Grip of robotic hand mimics human hand for handling different objects. The aim is to develop robotic hand handling of object similar to real human hand. The robotic hand design has 5 fingers 14 degree of freedom.
\end{abstract}

Keywords - Camera, Human-Robot Interaction, Gripper, Gesture, Grasping and Robotic Arm

\section{INTRODUCTION}

Robotics and robotic Arm plays vital role in the industries. Robots handles the products with the help of sensors such as acceleration sensor, EMG sensor, Gyroscope, pressure sensor, Tactile sensor, Tilt sensor strain gauge, Load cell, Proximity sensor, IR sensor, Ultrasonic sensor, Force Torque sensor, RGB color sensor [1] and [7].

The development of Vision based sensors at present achieves good performance of robotics using RGB camera, Kinet sensor, Oculus Rift, Leap Motion Controller. Human Robot interaction by using human hand gesture is important for exact control of robotics [25].Real time human hand control is used in some precious applications like surgery, nuclear power plant, disaster rescue, military, space research centre, and transmission line work. Preprogrammed robot cannot adopt with hazardous places. Some kind of situation needs exact human control where human does not enter into the hazardous place [4] and [6].

The virtual diagnostic and treatments can be done by the robotic hand which mimics the physical moments of doctor. The virtual surgical practice transform into robotics surgery such as virtual diagnostic and treatments [23] and [26].

The elderly peoples are difficult to handle daily activities due to hand grip strength problem. The loss of grip strength is a neurological hand disorder affecting the hand functionalities of people and patients [18].

Received: August 31, 2019

Reviewed: February 3, 2020

Accepted: March 20, 2020 
HRI (human robot interaction) uses many gesture sensors to control robotic hand. The flex sensors based hand gloves are used to measure human hand gesture in simplest and easy method to control robotic hand [19].

Image based gesture recognition provides better accuracy under proper lighting conditions. Image and vision based approach uses pre stored database. These types of methods are requires more memory space for database [20]. The interaction between human and robotic arm based on 3D vision system. The stereo camera used to track human hand signal as 3D scene and image. Vision sensor tracking also used tracking human intensions and grasping moving objects [21]. The human intensions can be measured by EMG pattern recognition to control myoelectric hand. EMG pattern recognition used for limb deficient people to perform daily activities [22].

\section{RELATED WORK}

The main problem of gesture controlled robotic arm is accuracy. Industrial robots are pre programmed or feedback based control [16]. The sensor glove measuring finger motion [2]. This sensor glove concept measures angle of the fingers [5].

Every one can communicate with gesture in day to day life. At some kind of situation not only the children, deaf and dump person, illiterate persons are doing gesture. The concept of gesture interaction between human and machine is to convert gesture movements into electrical signal using sensors. Electrical signals are measured from the sensor manipulated by computer or embedded processor to understand the useful information [9] and [17].

Gesture is an activity perceived by the flex sensor which is an analog form of signal. This analog signal is converted into digital signal to communicate with digital machine [3].

Soft robotic hand is developed and utilized in some industries. The design of soft robotic hand is based on the shape and size of the object [16]. The advantages of this type of robotic hands are flexibility and adoptability for handling objects. Capacitive touch sensor, rubber and suction type of materials are used in this soft grippers [8], and [10].

EMG sensor is used to measures electrical activities of human muscles for controlling robotic gripper for handling substance [11] and [12].

The development of visual sensors interface like camera. converts the real environment into virtual environment and further virtual environment into robotic environment to mimic as human hand gesture [14].

The teleoperation based free hand gesture or wearable hand gesture recognition requires feedback of sometimes to complete the object manipulation task. Information of human hand gesture acuired by vision based sensor such as camera. The gesture information send to remote site computer which process the received gesture information and control the robotic arm to perform like a human operator. The robot manipulator can be controlled by three methods such as direct control,tradded control and shared control by teleoperation. The goal of haptic feedback system increesing the human robot collabaration for the shared task [24].

\section{PROPOSED WORK}

Proposed system aims for exact control of robotic arm to achieve better accuracy using measurement of both human hand and robotic hand. This paper presents an implementation of flex sensor for robotic arm control with low cost [7] and [15]. The advantages are low cost and less hardware complexity. Both human hand and robotic hand measurement is used wherever high precisions are needed. 


\subsection{REAL TIME HUMAN HAND GRIP MEASUREMENT}

The flex sensor is the primary input of PIC16F877A microcontroller placed in human hand of five fingers to measure the deviation of the fingers. Five distinct flex sensors are connected in five fingers of human hand by using hand glove. The output of each flex sensors is fed into the ADC (analog to digital converter) channels of PIC16F877A microcontroller.

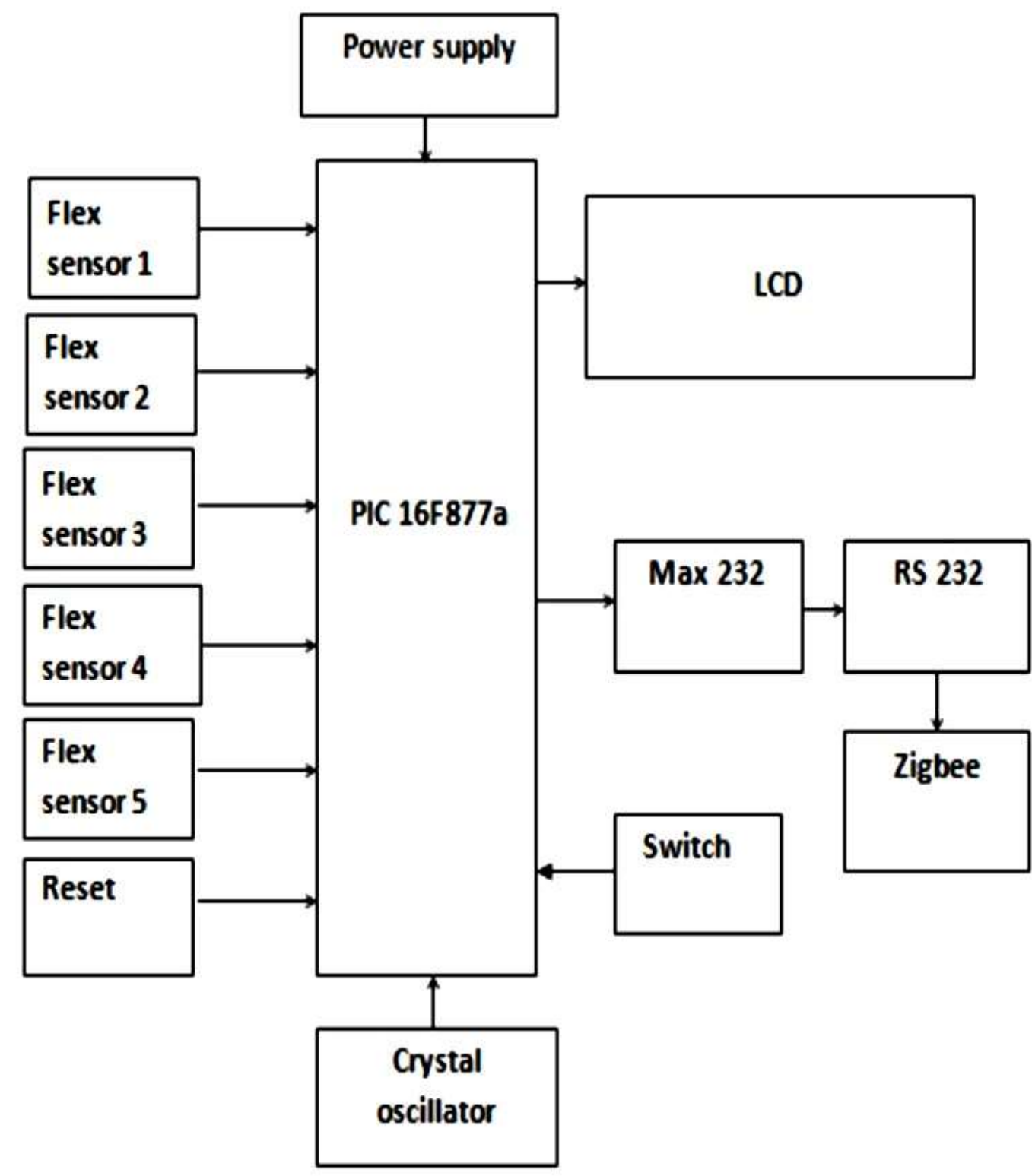

Fig. 1 Human Hand Gesture Measurement

Fig. 1 shows human hand gesture measurement using flex sensor. PIC16F877A microcontroller has built in 8 channel successive approximation type ADC with 10 bit resolution. The measured value of each channel of ADC values displays through the 2 line 16 character LCD. Max232 IC is a voltage level converter IC used to communicate via RS232 Port. Zigbee wireless device connected in RS232 port is to transmit measured values of flex sensors to robotic arm placed in the remote area. When the threshold values of particular hand gesture reached, then user can press the threshold switch to transmit 
useful gesture information. Program Code is written in "Embedded C" language using MPLAB IDE with cross compiler HI-TECH C PRO for the Pic10/12/16 MCU family.

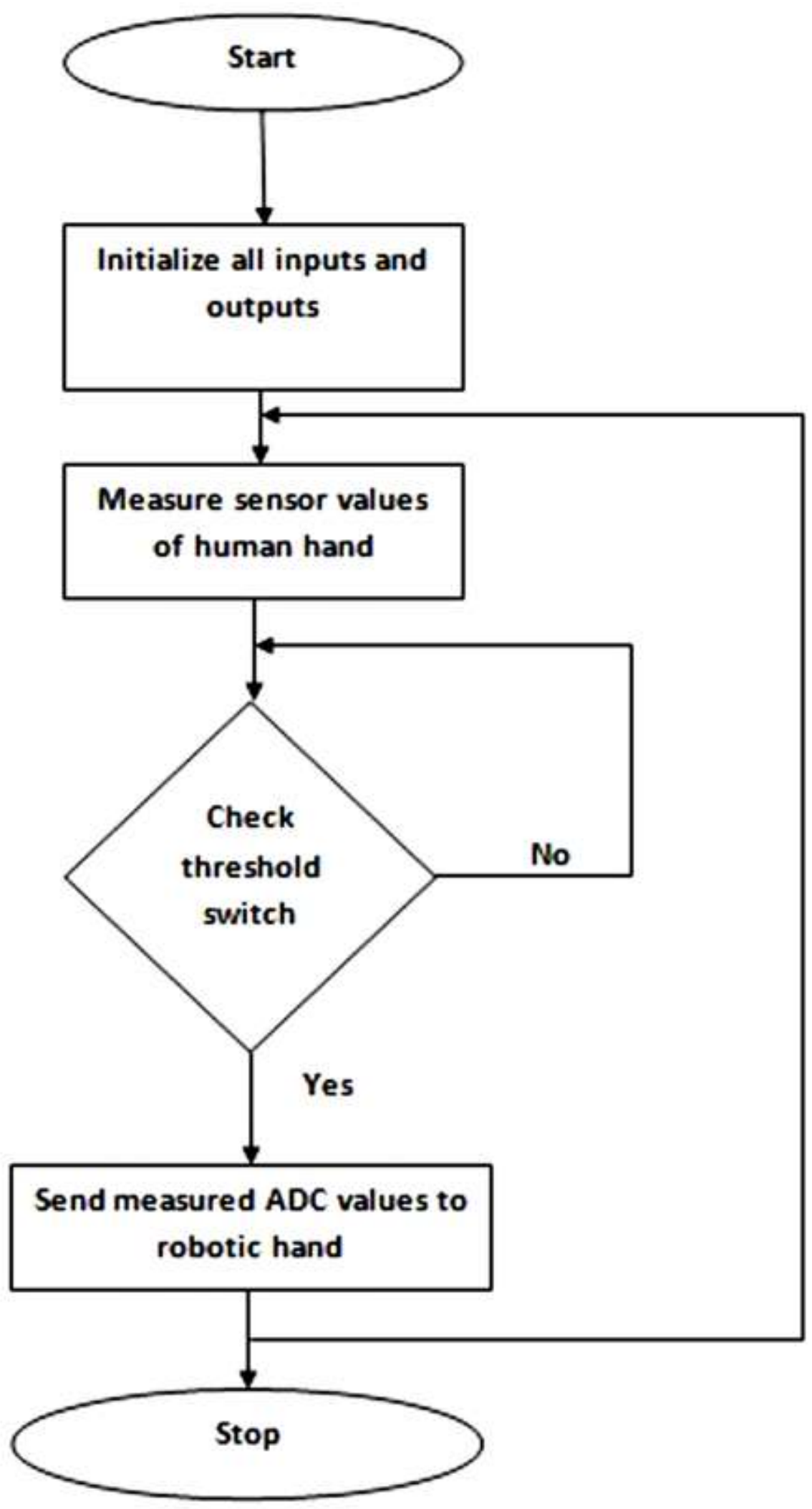

Fig. 2 Human hand gesture conversion process 
Fig. 2 shows the conversion process of human hand gesture. In this human hand gesture conversion process first step is to initialize all the inputs (sensors, switch) outputs (LCD) and UART communication used by the controller. Next step is sensor values measured by the analog to digital converter.

\subsection{ROBOTIC HAND GRIP MEASUREMENT}

The flex sensor is placed in Robotic hand of five fingers to measure the deviation of the fingers of robotic hand.

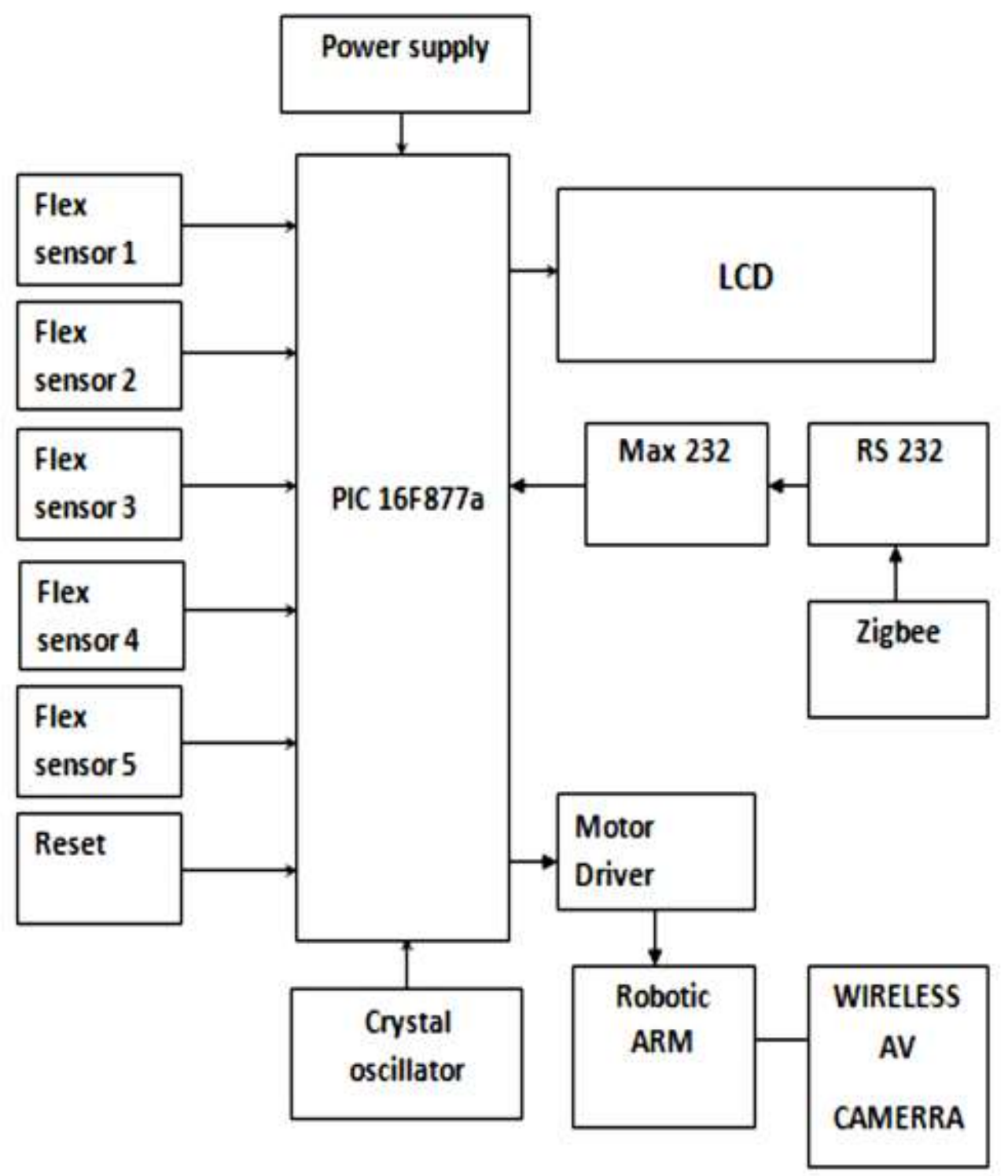

Fig. 3 Robotic Hand Gesture Measurement

Zigbee receives the hand gesture values of human hand from the control area. It compares the received hand gesture value with measured hand gesture value of robotic hand. When the received values and measured values are same then the robotic arm completes the task. 


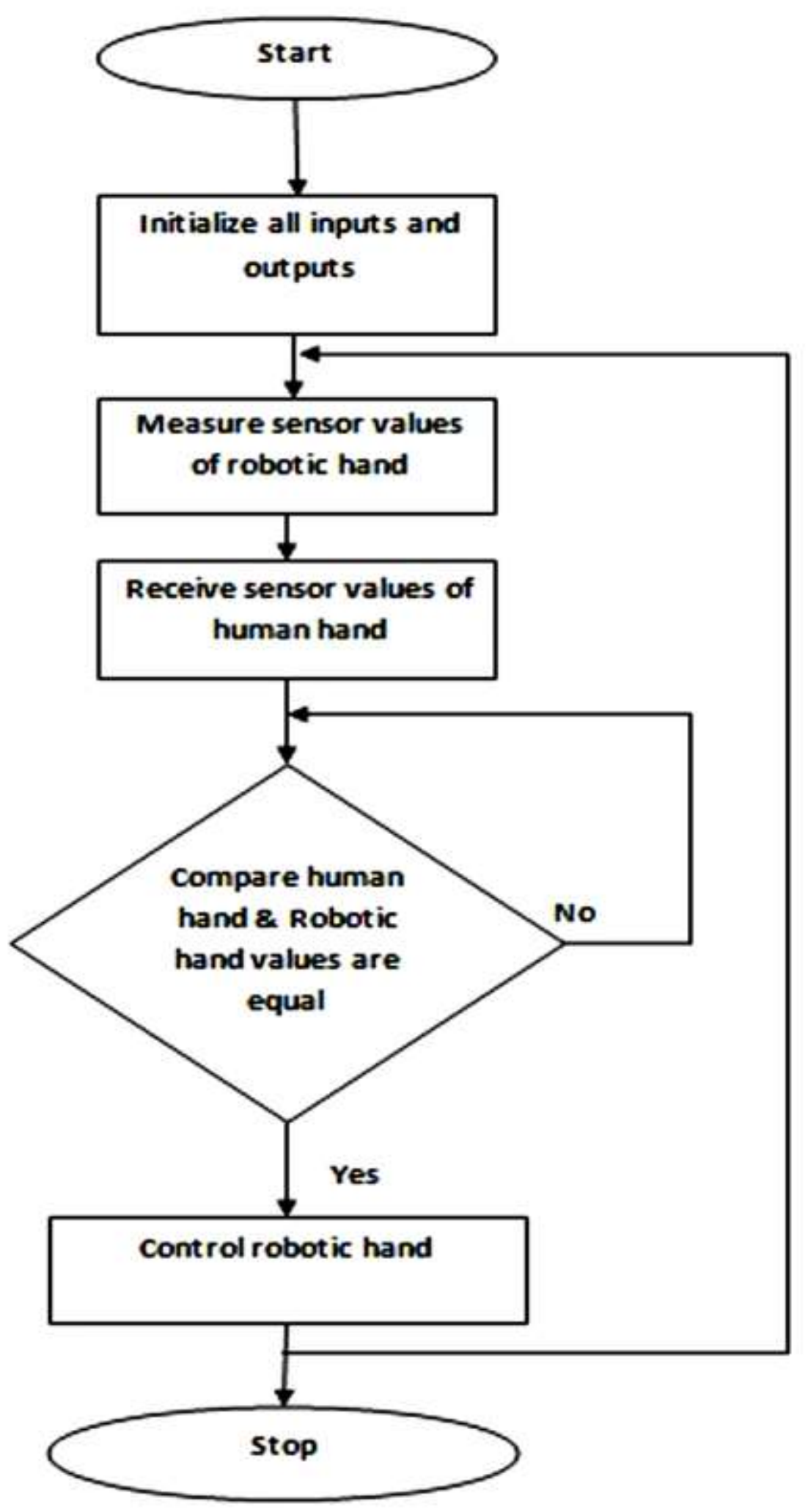

Fig. 4 Robotic Hand Gesture Control Process

Five distinct flex sensors are connected in five fingers of robotic hand by using hand glove. The output of each flex is sensors fed into the ADC channels of PIC16F877A 
microcontroller. Fig. 4 shows the measurement of robotic hand in accordance with human hand gesture.

\subsection{FLEX SENSOR}

The working of flex sensor is based on the values of the resistance which varies, as the body of sensor bends from normal position. The amount of deflection is directly proportional to resistance.

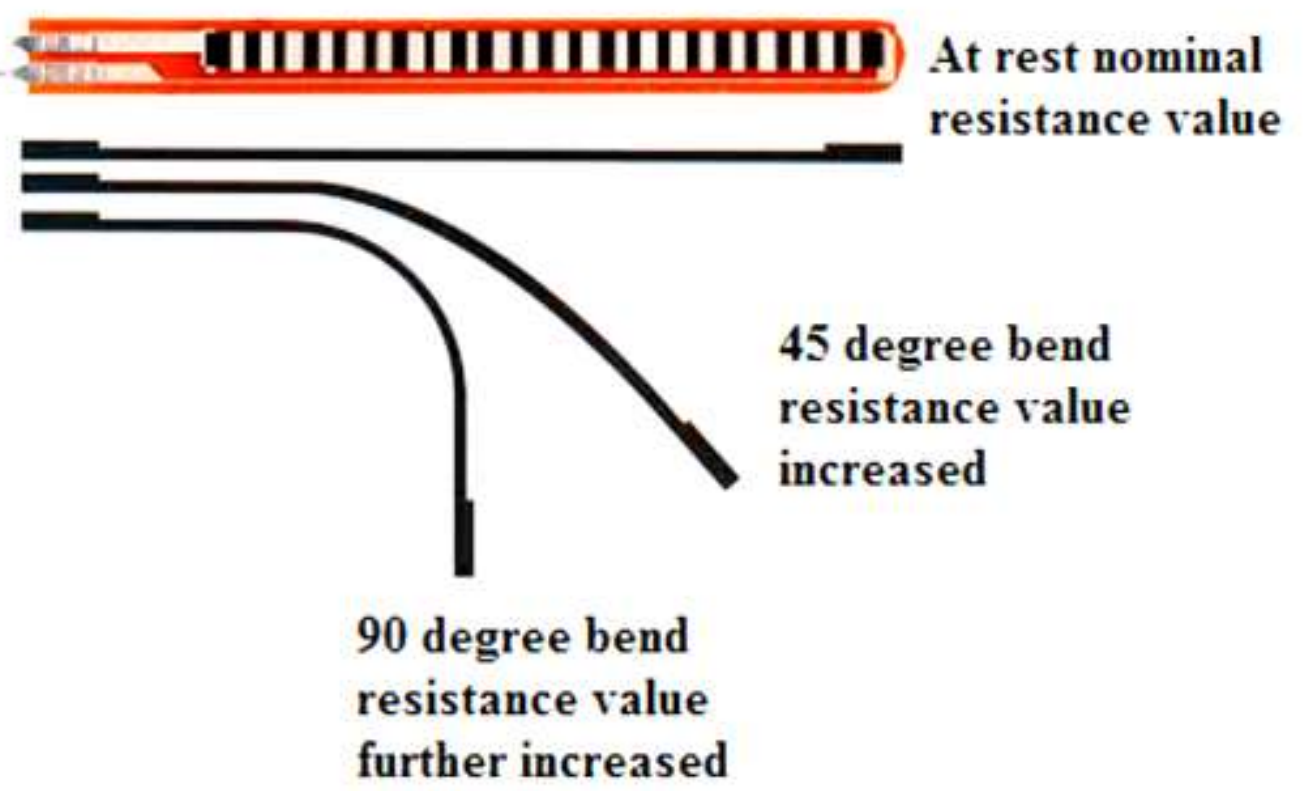

Fig. 5 Flex Sensor and Deflection

Fig. 5 shows the deflection of flex sensor and its resistance variations at degree of beddings.

\subsection{HAND SKELETAL}

Fig. 6 shows the skeletal view of human hand. The phalanges, metacarpals and carpals forms finger of human hand.

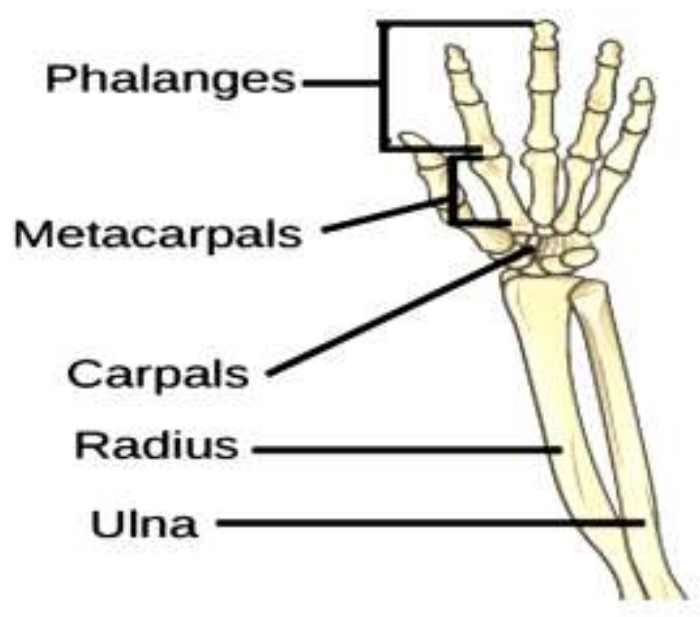

Fig. 6 Skeletal View of Human Hand 
The ulna and radius located between elbow and wrist joint are called as forearm. Five fingers consist of 14 bones. Palm formed by 5 bones and wrist contains 8 bones.

\subsection{ROBOTIC HAND DESIGN}

The design of robotic hand has five fingers. Each finger has separate servo motor to control robotic arm. Fig. 7(b) shows Servo motor can control three links and joints (distal, phalanges, metacarpals) of robotic hand. The design of robotic hand operates with 14 degree of freedom (DOF). Thumb finger has 2 DOF and residual each finger has 3 DOF.

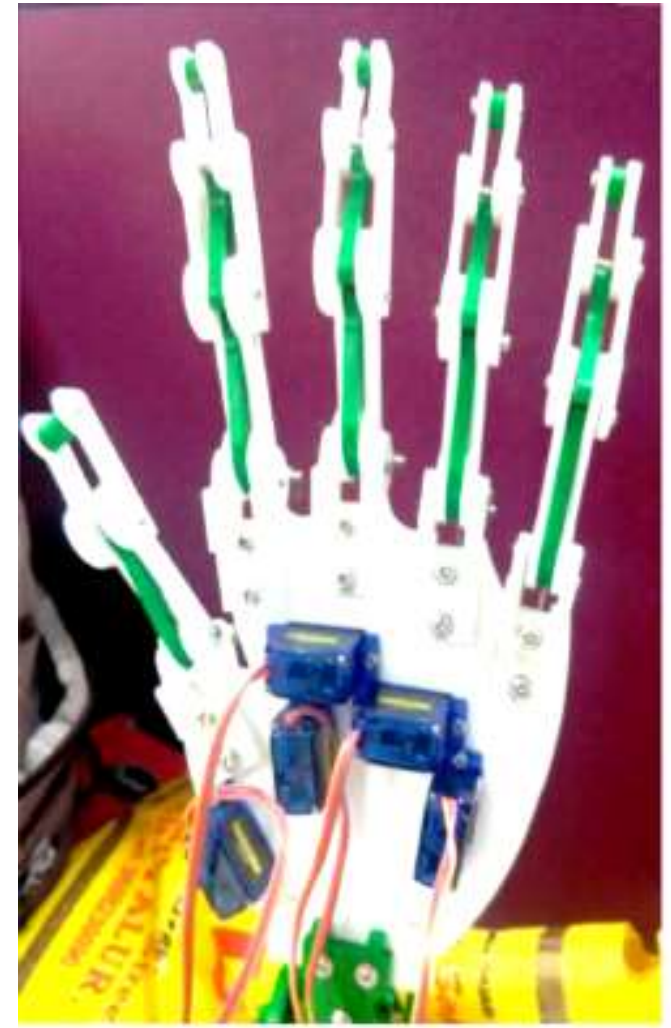

a)

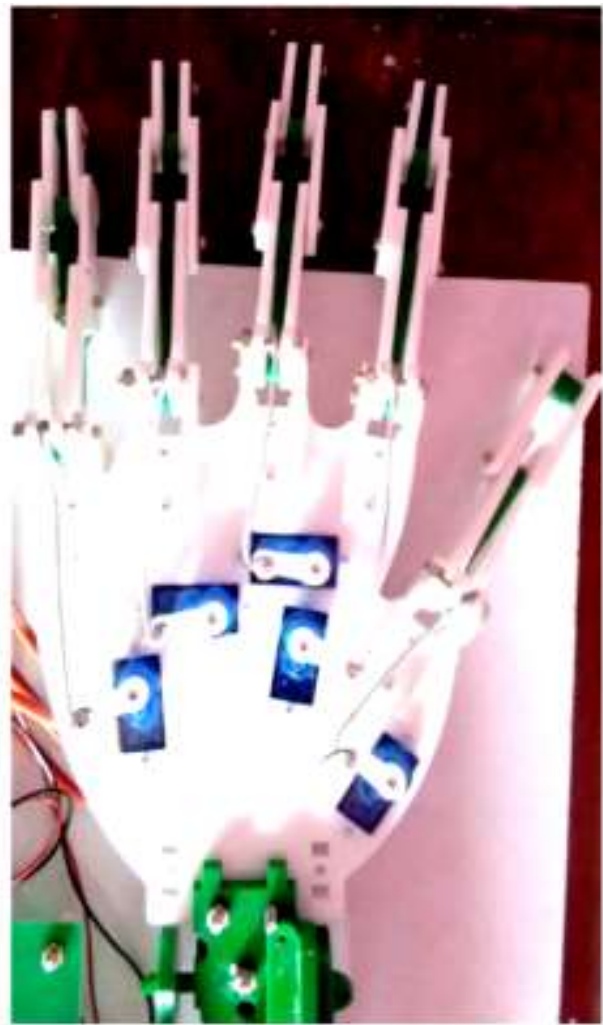

(b)

Fig. 7 Robotic arm (a) front view (b) back view

Fig. 7(a) shows five servo motor to control fingers of robotic hand arranged in the palm of the robotic hand. Fig. 7 shows the design of robotic hand similar to the human hand skeletal view.

\section{KINEMATICS OF ROBOTIC FINGER}

Fingers of robotic hand have three joints and thumb finger only has two joints. Fig. 8 shows kinematics of robotic finger.

$\theta_{1}$ angle of link $1\left(l_{1}\right), \theta_{2}$ angle of link $2\left(l_{2}\right), \theta_{3}$ angle of link $3\left(l_{3}\right)$ 

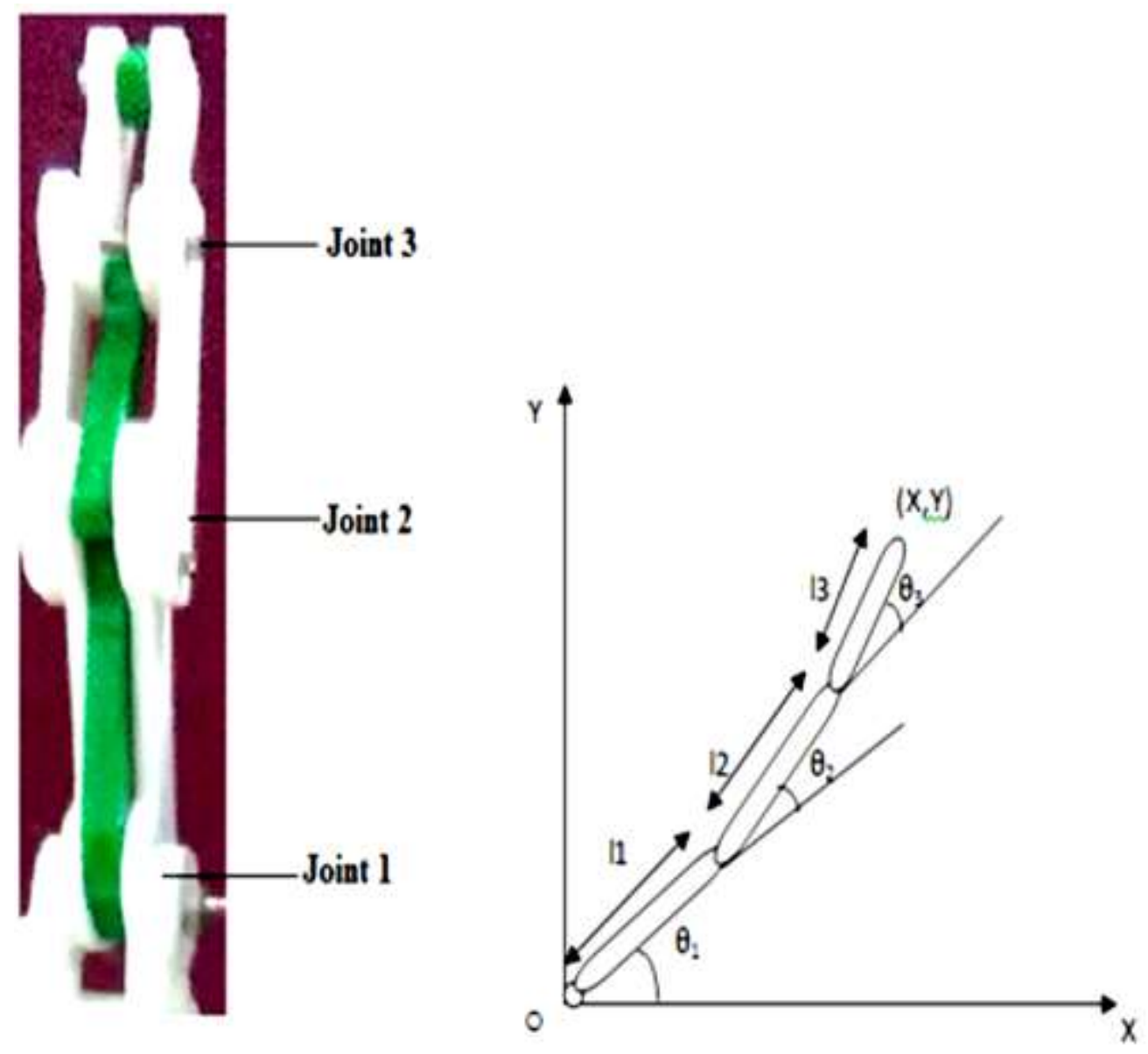

Fig. 8 Kinematics of Robotic Finger

Position of forward Kinematics and inverse Kinematics

Position of 1 st link is given by

$$
P 1\left(\theta_{1}\right)=\left(\begin{array}{ccc}
\operatorname{Cos}\left(\theta_{1}\right) & -\operatorname{Sin}\left(\theta_{1}\right) & 0 \\
\operatorname{Sin}\left(\theta_{1}\right) & \operatorname{Cos}\left(\theta_{1}\right) & 0 \\
0 & 0 & 1
\end{array}\right)
$$

Position of 2nd link is given by

$$
P 2\left(\theta_{2}\right)=\left(\begin{array}{ccc}
\operatorname{Cos}\left(\theta_{2}\right) & -\operatorname{Sin}\left(\theta_{2}\right) & l_{1} \\
\operatorname{Sin}\left(\theta_{2}\right) & \operatorname{Cos}\left(\theta_{2}\right) & 0 \\
0 & 0 & 1
\end{array}\right)
$$

Position of 3rd link is given by

$$
P 3\left(\theta_{a}\right)=\left(\begin{array}{ccc}
\operatorname{Cos}\left(\theta_{a}\right) & -\operatorname{Sin}\left(\theta_{a}\right) & l_{2} \\
\operatorname{Sin}\left(\theta_{a}\right) & \operatorname{Cos}\left(\theta_{a}\right) & 0 \\
0 & 0 & 1
\end{array}\right)
$$

Let $\omega=\theta 1+\theta 2+\theta 3$ since $C 123=C \omega, S 123=S \omega$, The solution for the forward kinematic problem is given by

$$
{ }_{0}^{4} P\left(\theta_{1}, \theta_{2}, \theta_{a}\right)=\left(\begin{array}{cccc}
C_{12 a} & -S_{12 a} & 0 & l_{1} C_{1}+l_{2} C_{12}+l_{a} C_{12 a} \\
S_{12 a} & C_{12 a} & 0 & l_{1} S_{1}+l_{2} S_{12}+l_{a} S_{12 a} \\
0 & 0 & 1 & 0 \\
0 & 0 & 0 & 1
\end{array}\right)
$$


Kinematic equation for 3 link manipulator

$$
\begin{aligned}
& X=l_{1} \operatorname{Cos}\left(\theta_{1}\right)+l_{2} \operatorname{Cos}\left(\theta_{1}+\theta_{2}\right)+l_{a} \operatorname{Cos}(\omega) \\
& Y=l_{1} \operatorname{Sin}\left(\theta_{1}\right)+l_{2} \operatorname{Sin}\left(\theta_{1}+\theta_{2}\right)+l_{2} \operatorname{Sin}(\omega)
\end{aligned}
$$

Position of link 1 is

$$
\begin{aligned}
& X_{1}=X-l_{2} \operatorname{Cos}\left(\theta_{1}+\theta_{2}\right)+l_{a} \operatorname{Cos}(\omega) \\
& Y_{1}=Y-l_{2} \sin \left(\theta_{1}+\theta_{2}\right)+l_{a} \sin (\omega)
\end{aligned}
$$

Position of link 2 is

$$
\begin{aligned}
& X_{2}=X-l_{a} \operatorname{Cos}(\omega) \\
& X_{2}=l_{1} C_{1}+l_{2} C_{12 a} \\
& Y_{2}=Y-l_{a} \operatorname{Sin}(\omega) \\
& Y_{2}=l_{1} S_{1}+l_{2} S_{12}
\end{aligned}
$$

Squaring both side of (10) \& (12)

$$
\begin{aligned}
& X_{2}^{2}+Y_{2}^{2}=l_{1}^{2}+l_{2}^{2}+2 l_{1} l_{2} C_{2} \\
& C_{2}=\frac{X_{2}^{2}+Y_{2}^{2}-l_{1}^{2}-l_{2}^{2}}{2 l_{1} l_{2}} \\
& \left.S_{2}= \pm \sqrt{\left(1-C_{2}^{2}\right.}\right) \\
& \theta_{2}=\operatorname{aTan} 2\left(S_{2}, C_{2}\right) \\
& X_{2}=\left(l_{1}+l_{2} C_{2}\right) C_{1}-l_{2} S_{2} S_{2} \\
& Y_{2}=\left(l_{1}+l_{2} C_{2}\right) S_{1}+l_{2} C_{1} S_{2} \\
& S_{1}=\frac{\left(l_{1}+l_{2} C_{2}\right) Y_{2}-l_{2} S_{2} X_{2}}{\Delta} \\
& C_{1}=\frac{\left(l_{1}+l_{2} C_{2}\right) X_{2}+l_{2} S_{2} Y_{2}}{\Delta} \\
& \Delta=l_{2}^{2}+l_{2}^{2}+2 l_{1} l_{2} C_{2}=X_{2}^{2}+Y_{2}^{2} \\
& \theta_{1}=\operatorname{aTan} 2\left(S_{1}, C_{1}\right) \\
& \theta_{a}=\omega-\theta_{1}-\theta_{2}
\end{aligned}
$$

\section{EXPERIMENTAL RESULT}

Fig. 9 shows the human hand with flex sensor for grip measurement and PIC microcontroller with LCD which shows the each fingers gesture ADC values on the display. 


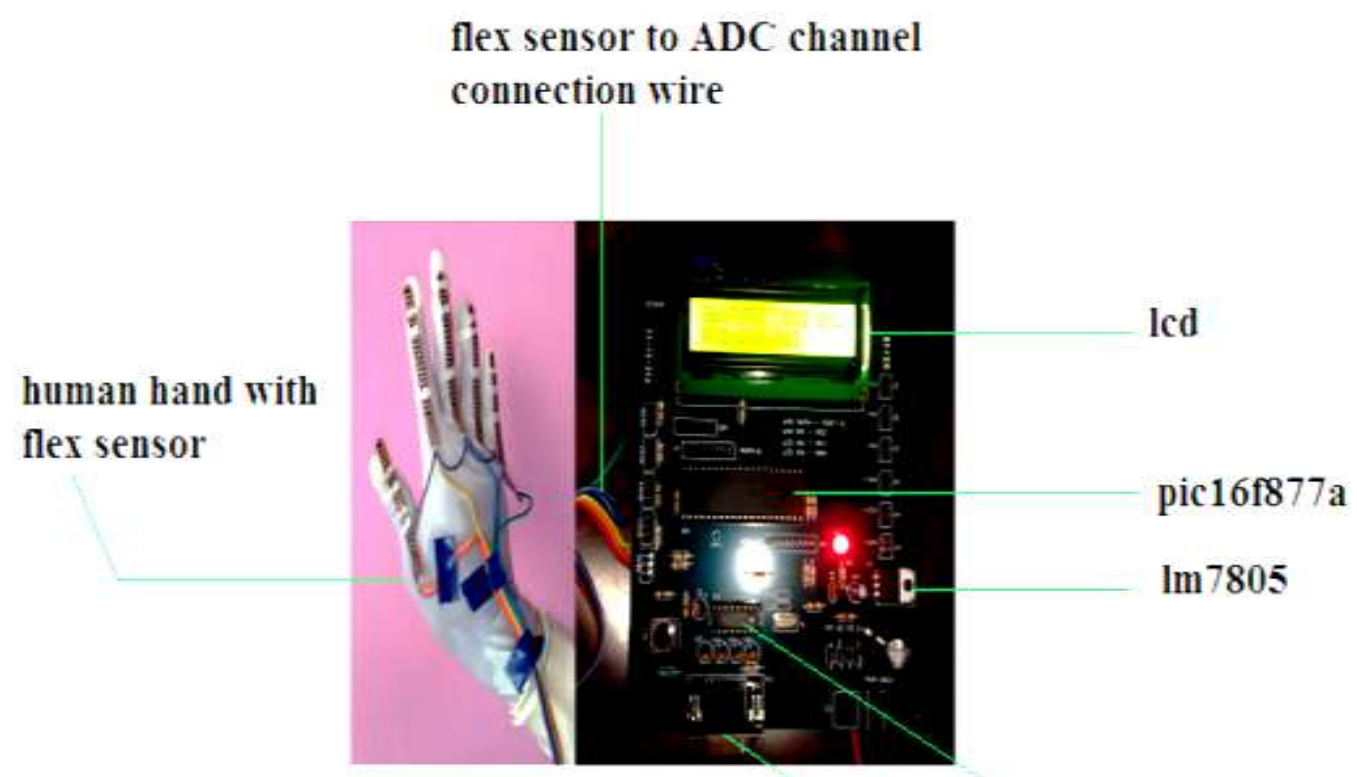

$\operatorname{Max} 232$

\section{RS232}

connector

Fig. 9 Human Hand Gesture Measured Value on LCD

This ADC values may be coded for different hand gesture such as grab, close, release, and pinch for robotic hand. The values measured by flex sensor in human hand gloves Shows Table I.

Table I. Human Hand Gesture Measured Values for each Finger

\begin{tabular}{|l|l|l|l|l|l|}
\hline Gestures & \multicolumn{4}{|l|}{$\begin{array}{l}\text { Measured human hand fingers gesture } \\
\text { value (decimal) }\end{array}$} \\
\cline { 2 - 6 } & Thump & Index & Middle & Ring & Pinky \\
\hline open & 12 & 12 & 12 & 12 & 12 \\
\hline close & 09 & 09 & 09 & 09 & 09 \\
\hline pinch & 11 & 10 & 12 & 12 & 12 \\
\hline Grab & 09 & 09 & 08 & 09 & 09 \\
\hline
\end{tabular}

Fig. 10 shows flex sensor values for each human finger at various hand gestures such as open, close, pinch, grab. 


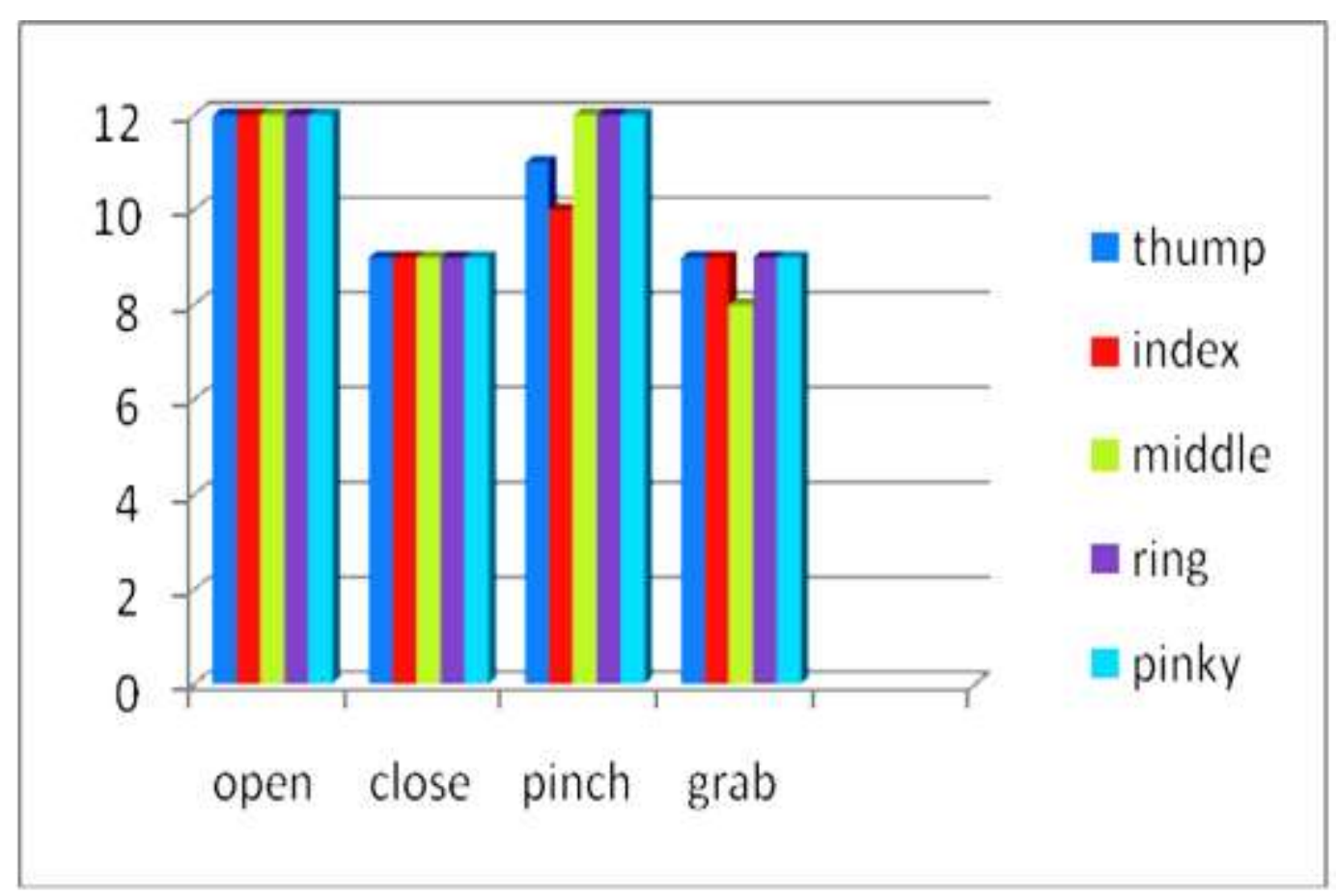

Fig. 10 Human Hand Gestures and its Sensor Values

Fig. 11 shows the robotic hand with flex sensor for grip measurement and PIC microcontroller with LCD which shows the each fingers gesture of ADC values on the display.

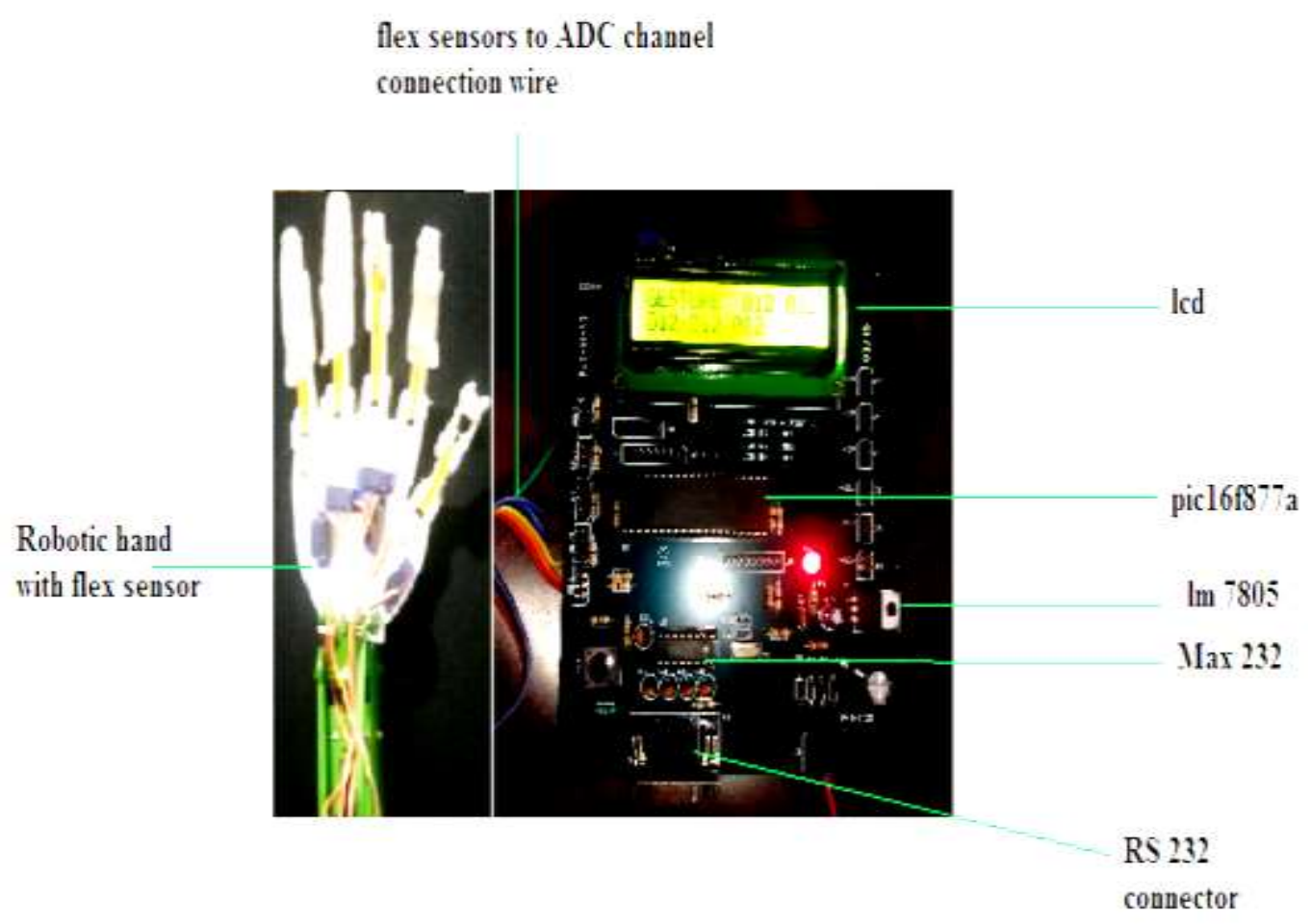

Fig. 11 Robotic Hand Gesture Measured Value on LCD 
These ADC values of robotic hand and the ADC values of human hand is compared for different hand gestures such as grab, close, release, pinch for robotic hand. When these two comparison values are equal then it improve the performance accuracy.

Table II. Robotic hand gesture measured value for each finger

\begin{tabular}{|l|l|l|l|l|l|}
\hline \multirow{2}{*}{ Gestures } & \multicolumn{5}{|l|}{ Measured robotic hand fingers gesture value (decimal) } \\
\cline { 2 - 6 } & Thump & Index & Middle & Ring & Pinky \\
\hline open & 12 & 12 & 12 & 12 & 12 \\
\hline close & 10 & 09 & 09 & 09 & 09 \\
\hline pinch & 10 & 10 & 12 & 12 & 12 \\
\hline Grab & 09 & 09 & 08 & 08 & 09 \\
\hline
\end{tabular}

The robotic arm gesture values of each fingers measured by flex sensor. The sensor values measured at robotic hand shows Table II.

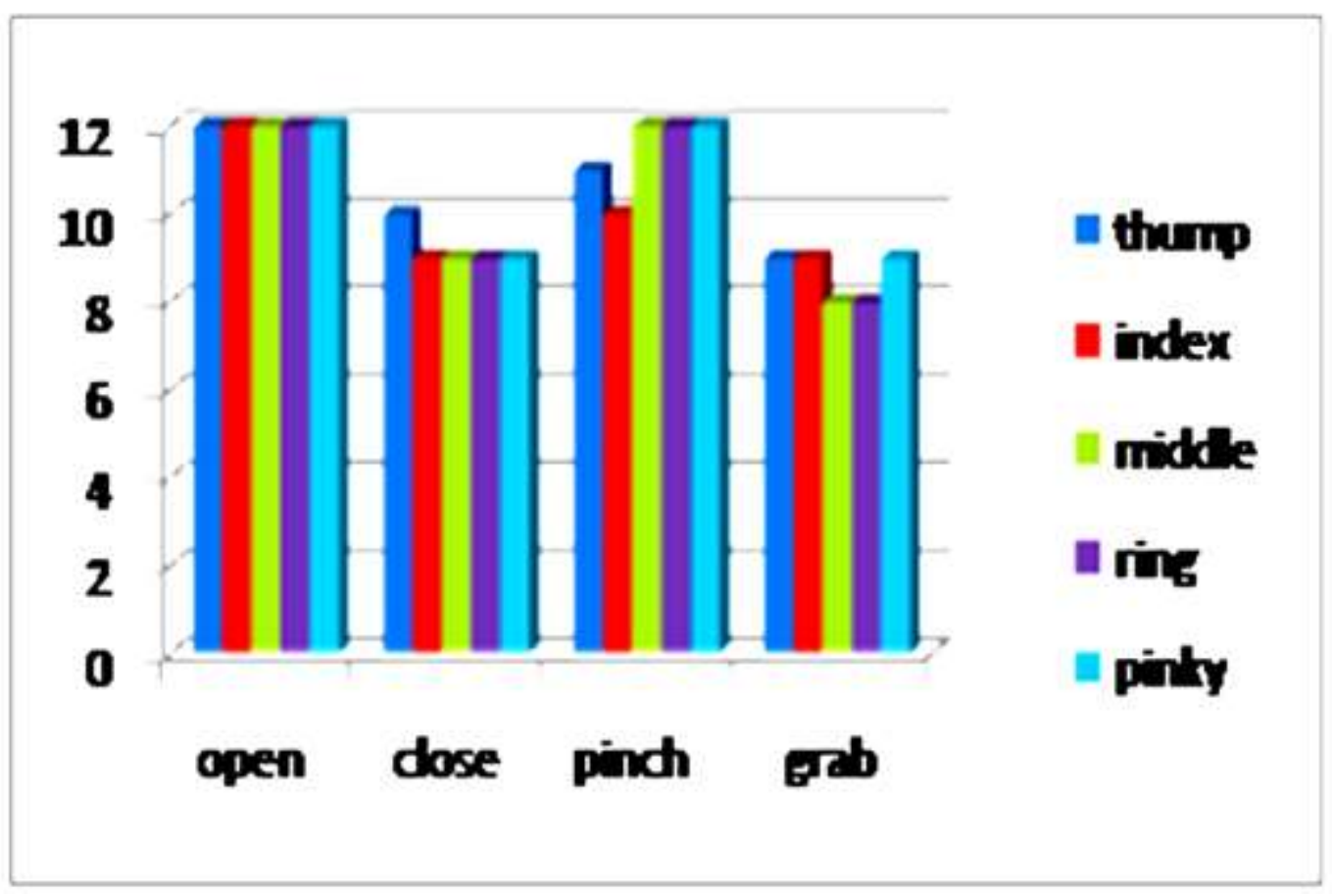

Fig. 12 Robotic Hand Gesture and its Sensor Values

Fig. 12 shows flex sensor values of each fingers of robotic hand at various hand gestures such as open, close, pinch, and grab. 


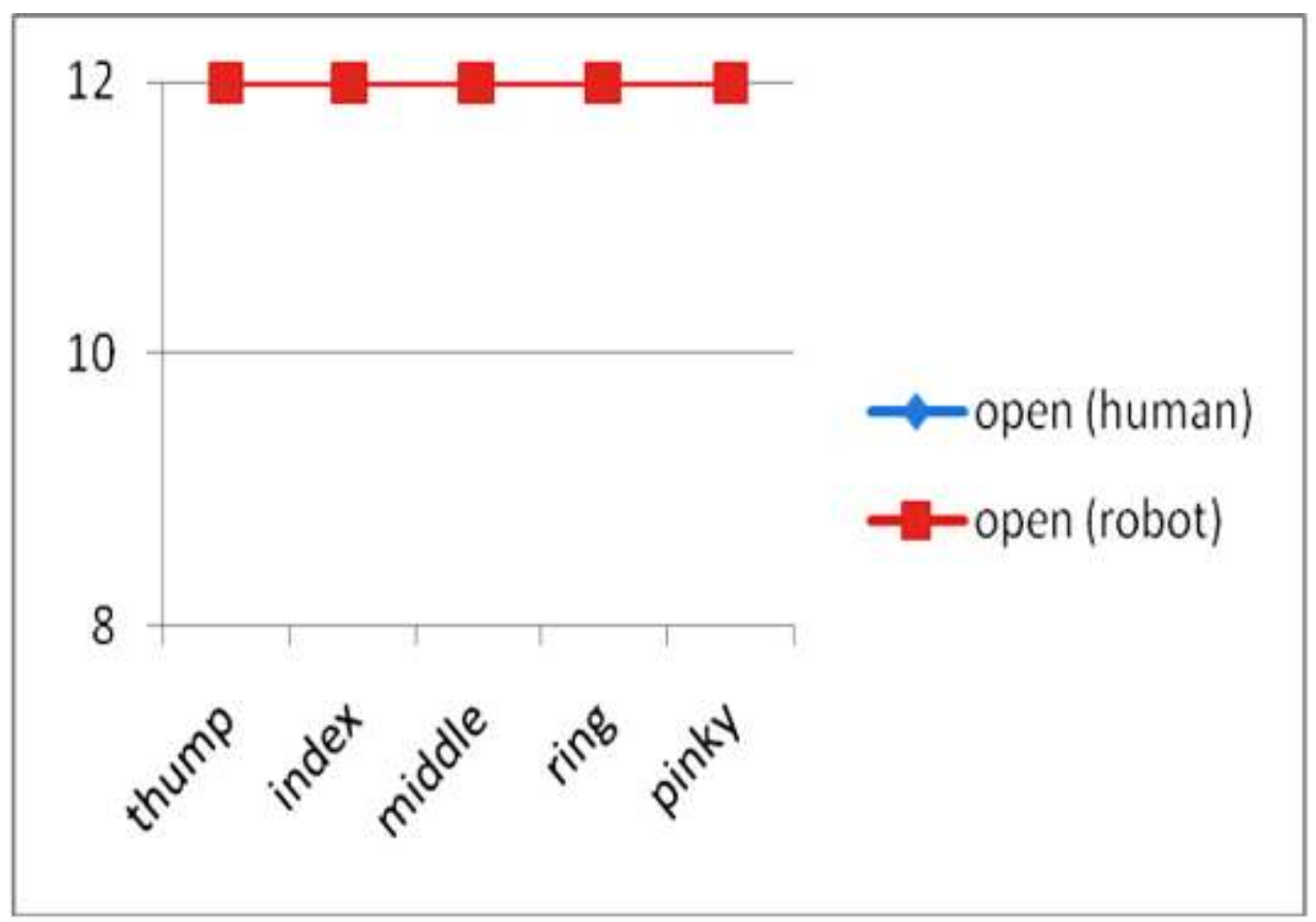

(a) Comparison of human hand and robotic hand open gesture

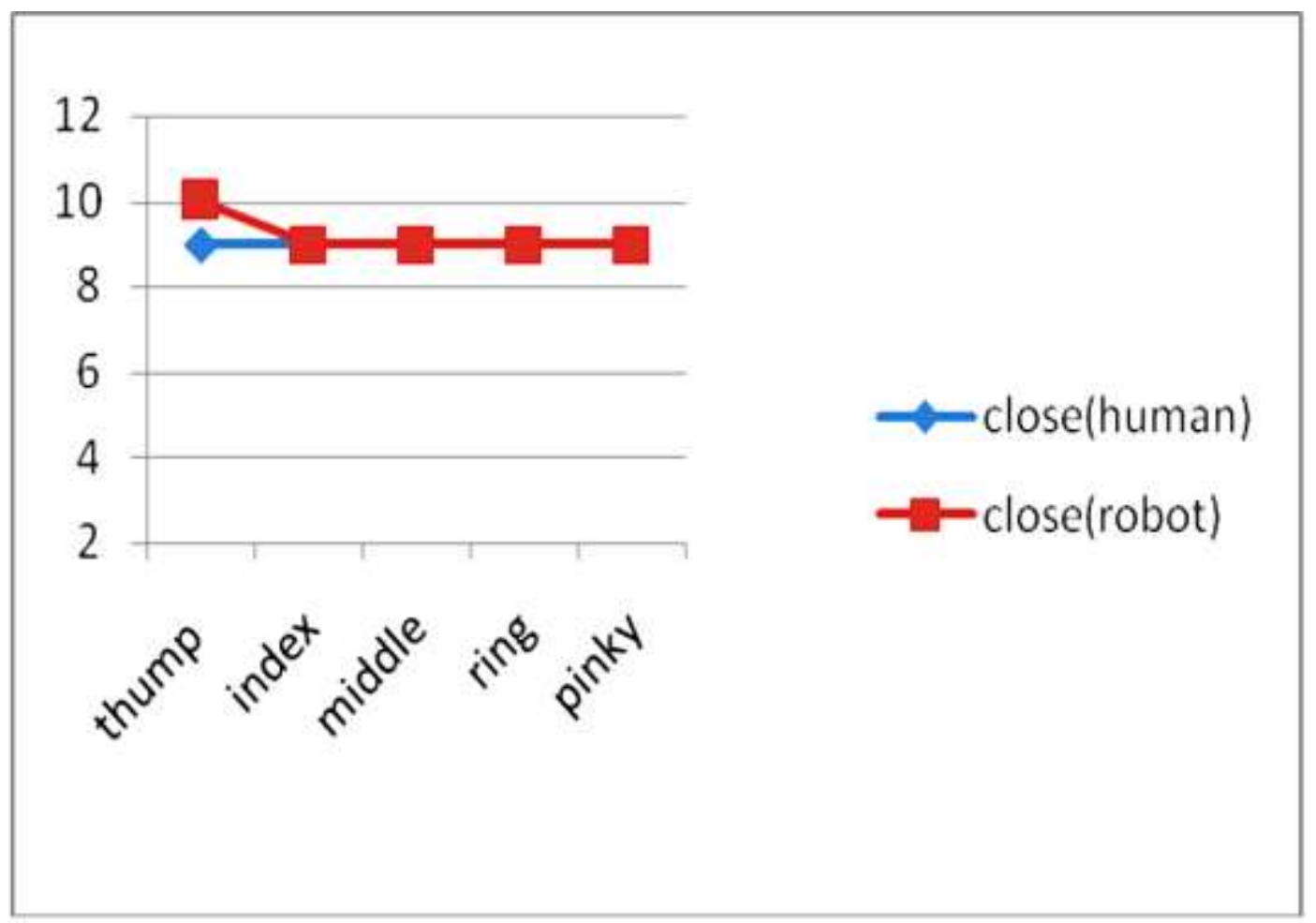

(b) Comparison of human hand and robotic hand close gesture 


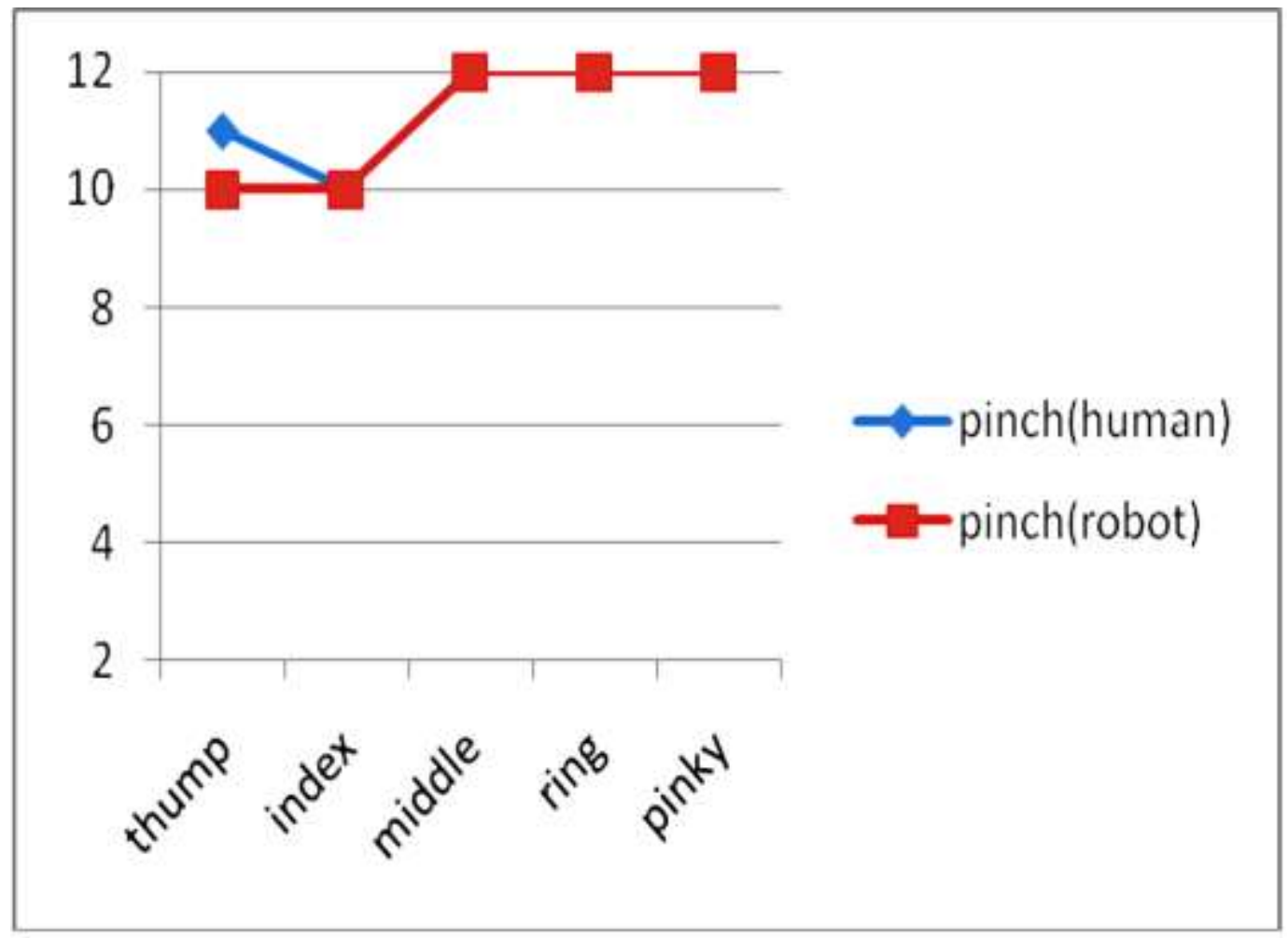

(c) Comparison of human hand and robotic hand pinch gesture

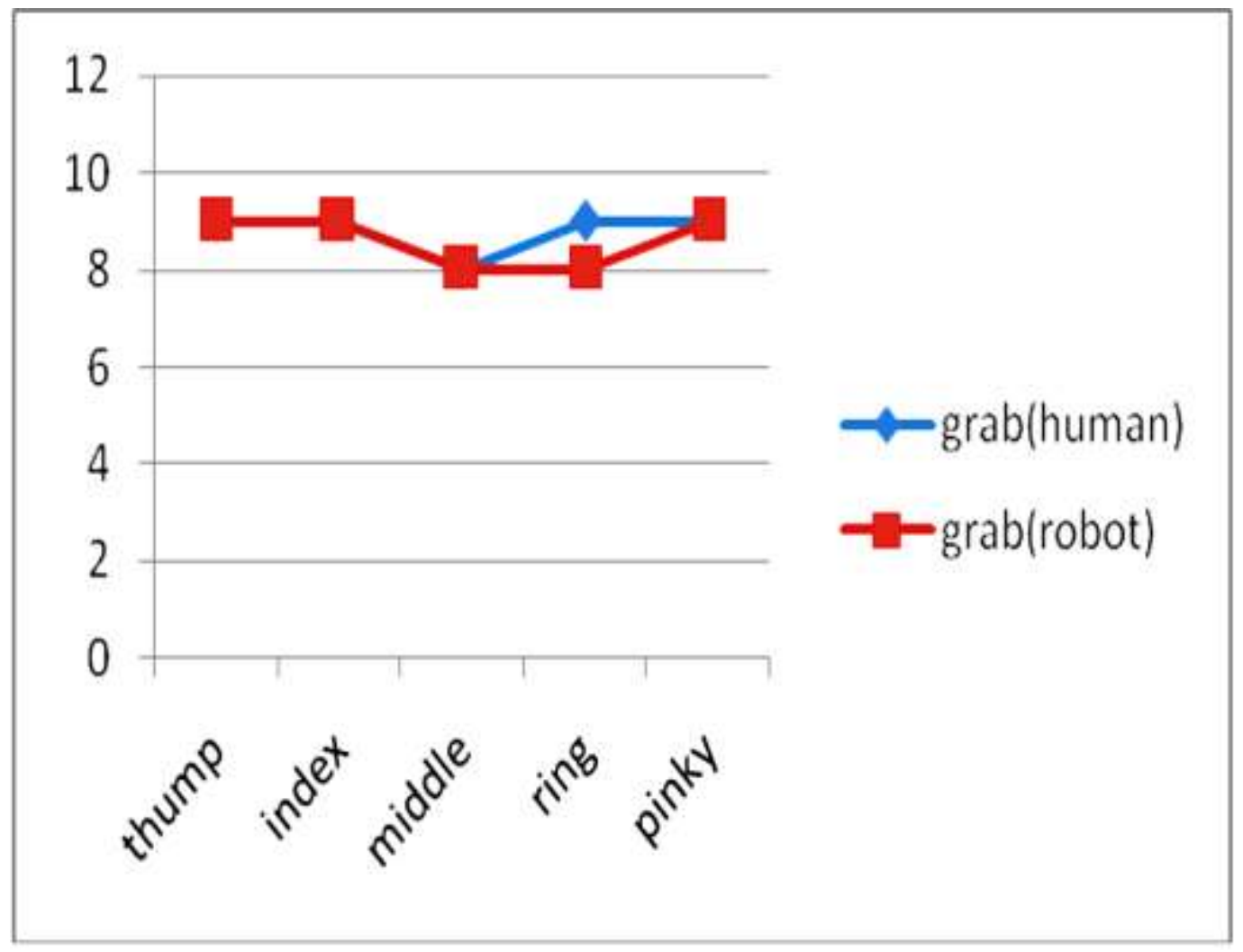

(d) Comparison of human hand and robotic hand grab gesture

Fig. 13 Comparison of Human Hand and Robotic Hand Gestures 
Fig. 13 shows the comparison result of human hand gestures and robotic hand gestures. Fig. 13(a) shows open gesture of human hand and robotic hand, Fig. 13(b) shows close gesture of human hand and robotic hand, Fig. 13(c) shows pinch gesture of human hand and robotic hand and Fig. 13(d) shows grab gesture of human hand and robotic hand.

\section{DISCUSION}

Both end measurement uses same sensors on both human and robotic hand since it produce same values for the same deflection of flex sensor at both end. When the both end measured ADC output value same then it achieves the exact control. The development of vision based sensors are detects gesture also produce vision and good accuracy with high cost. The proposed method of both end measurement produce good accuracy with affordable cost.

Robotic hand developed for testing such as human hand. It has 14 DOF. Thumb finger has $2 \mathrm{DOF}$ and each other finger has 3 links with separate servo motor to control finger movement. This robotic hand model produces high accuracy for close (pick), open (place) and grab. This design is well suitable for pick and place related applications.

\section{CONCLUSION}

In this research work, the proposed concept of controlling robotic arm using both human and robotic hand measurements ware performed with better accuracy. Both end measurement goes high precision for handling task by robotic arm.

The pick and place is the major operation in industries. So, this method produces better accuracy for open and close hand gesture. This paper measure the cooperation of human hand and robotic hand. The design of same sensor based hand gloves used at both end to measure the human and robotic hand performance and task cooperation. Many visual gesture sensors available present days but not affordable since this both end measurement method is better for exact control with low cost solutions.

\section{ACKNOWLEDGMENTS}

Author would like to thank Fliptronics Research and Development Laboratory for the support of experimental contribution of robotic hand design and testing.

\section{REFERENCES}

[1] Saudabayev, Artur, and Huseyin Atakan Varol. "Sensors for robotic hands:A survey of state of the art." IEEE Access 3 (2015): 1765-1782.

[2] Park, Yeongyu, Jeongsoo Lee, and Joonbum Bae. "Development of a wearable sensing glove for measuring the motion of fingers using linear potentiometers and flexible wires." IEEE Transactions on Industria Informatics 11.1 (2014): 198-206.

[3] Tabassum, Meher, and D. D. Ray. "Intuitive control of three fingers robotic Gripper with a Data hand glove." 2013 International Conference on Control, Automation, Robotics and Embedded Systems (CARE). IEEE,2013.

[4] Wang, Zhongkui, Yuuki Torigoe, and Shinichi Hirai. "A prestressed soft gripper: design, modeling, fabrication, and tests for food handling." IEEE Robotics and Automation Letters 2.4 (2017): 1909-1916.

[5] Kawaguchi J, Yoshimoto S, Kuroda Y, Oshiro O. Estimation of finger joint angles based on electromechanical sensing of wrist shape. IEEE Transactions on Neural Systems and Rehabilitation Engineering. 2016 Nov 9;25(9):1409-18.

[6] Zhakypov Z, Heremans F, Billard A, Paik J. An origami-inspired reconfigurable suction gripper for picking objects with variable shape and size. IEEE Robotics and Automation Letters. 2018 Jun 14;3(4):2894-901.

[7] Sato K, Kamiyama K, Kawakami N, Tachi S. Finger-shaped gelforce: sensor for measuring surface traction fields for robotic hand. IEEE Transactions on Haptics. 2009 Oct 30;3(1):37-47.

[8] Tavakoli M, Lopes P, Lourenco J, Rocha RP, Giliberto L, de Almeida AT, Majidi C. Autonomous selection of closing posture of a robotic hand through embodied soft matter capacitive sensors. IEEE Sensors Journal. 2017 Jul 14;17(17):5669-77. 
[9] Purohit, Ankit, and Makarand Kakatkar. "A 9-dof robotic hand Teleoperation system using haptic technology." 2015 International Conference on Pervasive Computing (ICPC). IEEE, 2015.

[10] Ohka, Masahiro, Yuki Aiba, and Yusuke Motegi. "Assembly task based on tri-axial tactile data using loose grip." 2017 IEEE International Symposium on Robotics and Intelligent Sensors (IRIS). IEEE, 2017.

[11] Mangukiya, Yamik, Brij Purohit, and Kiran George. "Electromyography (EMG) sensor controlled assistive orthotic robotic arm for forearm movement." 2017 IEEE Sensors Applications Symposium (SAS). IEEE,2017.

[12] Leonardis D, Barsotti M, Loconsole C, Solazzi M, Troncossi M, Mazzotti C, Castelli VP, Procopio C, Lamola G, Chisari C, Bergamasco M. An EMG-controlled robotic hand exoskeleton for bilateral rehabilitation. IEEE transactions on haptics. 2015 Mar 30;8(2):140-51.

[13] Cho SG, Yoshikawa M, Baba K, Ogawa K, Takamatsu J, Ogasawara T. Hand motion recognition based on forearm deformation measured with a distance sensor array. In2016 38th Annual International Conference of the IEEE Engineering in Medicine and Biology Society (EMBC) 2016 Aug 16 (pp. 4955 4958). IEEE.

[14] Arata J, Hattori M, Ichikawa S, Sakaguchi M. Robotically enhanced rubber hand illusion. IEEE transactions on haptics. 2014 Feb 5;7(4):526-32.

[15] Hashimoto Y, Yatou D, Yamada T, Yamamoto H. Fundamental study on robotic assembly of modular fixture parts by a low cost 4-finger 12 dof hand. In2015 IEEE/SICE International Symposium on System Integration (SII) 2015 Dec 11 (pp. 930-935). IEEE.

[16] Low JH, Lee WW, Khin PM, Thakor NV, Kukreja SL, Ren HL, Yeow CH. Hybrid tele-manipulation system using a sensorized 3-D-printed soft robotic gripper and a soft fabric-based haptic glove. IEEE Robotics and Automation Letters. 2017 Jan 19;2(2):880-7.

[17] Naglot, Deepali, and Milind Kulkarni. "Real time sign language recognition using the leap motion controller." 2016 International Conference on Inventive Computation Technologies (ICICT). Vol. 3.IEEE, 2016.

[18] Ciullo, Andrea S., et al. "Analytical and experimental analysis for position optimization of a grasp assistance supernumerary robotic hand." IEEE Robotics and Automation Letters 3.4 (2018): 4305-4312.

[19] Sandesh, R. S., and Nithya Venkatesan. "Novel approach to control of robotic hand using flex sensors." IAES International Journal of Robotics and Automation 3.4 (2014): 234.

[20] Raheja, Jagdish Lal, et al. "Real-time robotic hand control using hand gestures." 2010 Second International Conference on Machine Learning and Computing. IEEE, 2010.

[21] Allen, Peter K., et al. "Automated tracking and grasping of a moving object with a robotic hand-eye system." IEEE Transactions on Robotics and Automation 9.2 (1993): 152-165.

[22] Chu, Jun-Uk, et al. "A supervised feature-projection-based real-time EMG pattern recognition for multifunction myoelectric hand control." IEEE/ASME Transactions on Mechatronics 12.3 (2007): 282290.

[23] Enayati, Nima, Elena De Momi, and Giancarlo Ferrigno. "Haptics in robot-assisted surgery: Challenges and benefits." IEEE reviews in biomedical engineering 9 (2016): 49-65.

[24] Kofman, Jonathan, et al. "Teleoperation of a robot manipulator using a vision-based human-robot interface." IEEE transactions on industrial electronics 52.5 (2005): 1206-1219.

[25] R.Satheeshkumar and R. Arivoli , "Real Time Virtual Human Hand For Robotics." Journal of Computational Information Systems 15.1 (2019): 82-89

[26] R.Satheeshkumar and R. Arivoli , "Real Time Virtual Human Hand for Diagnostic Robot (DiagBot) Arm Using IOT “ Journal of advanced research in dynamical and control system, Vol. 12, 01-Special Issue, 2020. 
International Journal of Advanced Science and Technology Vol.136 (2020) 